\title{
Identification, Expression and Co-Expression Analysis of R2R3-MYB Family Genes Involved in Graft Union Formation in Pecan (Carya illinoinensis)
}

\author{
Kaikai Zhu ${ }^{1,2} \mathbb{D}$, Pinghua Fan ${ }^{1,2}$, Zhenghai Mo ${ }^{3}$, Pengpeng Tan ${ }^{1,2}$, Gang Feng ${ }^{2}$, Fengda Li ${ }^{1,2}$ \\ and Fangren Peng 1,2,* \\ 1 Co-Innovation Center for Sustainable Forestry in Southern China, Nanjing Forestry University, \\ Nanjing 210037, China; kkzhu@njfu.edu.cn (K.Z.); 15295537732@163.com (P.F.); \\ tanpengpeng2002@163.com (P.T.); njfulifengda@163.com (F.L.) \\ 2 College of Forestry, Nanjing Forestry University, Nanjing 210037, China; pecanfeng@163.com \\ 3 Institute of Botany, Jiangsu Province and Chinese Academy of Sciences, Nanjing 210014, China; \\ mozhenghai@yeah.net \\ * Correspondence: frpeng@njfu.edu.cn; Tel.: +86-25-8542-7995
}

Received: 14 July 2020; Accepted: 19 August 2020; Published: 21 August 2020

\begin{abstract}
Plant R2R3-MYBs comprise one of the largest transcription factor families; however, few $R 2 R 3-M Y B$ genes in pecan have been functionally analyzed due to the limited genome information and potential functional redundancy caused by gene duplication. In this study, $153 R 2 R 3-M Y B$ genes were identified and subjected to comparative phylogenetic analysis with four other plant species. Then, the pecan R2R3-MYB gene family was divided into different clades, which were also supported by gene structure and motif composition results. Fifty-two duplication events including 77 R2R3-MYB genes were identified in this gene family, and $\mathrm{Ka} / \mathrm{Ks}$ values showed that all of the duplication events were under the influence of negative selection. Expression levels of pecan R2R3-MYB genes during the graft union formation process were further investigated using RNA-seq with four different timepoints after grafting, namely, 0, 8, 15 and $30 \mathrm{~d}$. Sixty-four differentially expressed R2R3-MYB genes were identified and showed different expression patterns after grafting. Co-expression networks were further constructed to discover the relationships between these genes. The co-expression networks contained 57 nodes (R2R3-MYB genes) and 219 edges (co-expression gene pairs) and CIL1528S0032 contained the maximum number of edges. Fifteen genes contained more than 10 edges; the majority of these were up-regulated during graft union formation and verified by qRT-PCR. This study provides a foundation for functional analysis to investigate the roles that R2R3-MYBs play in graft union formation in pecan and identify the key candidate genes.
\end{abstract}

Keywords: Carya illinoinensis (Wangenh.) K. Koch; gene expression; grafting; phylogenetic relationship; R2R3-MYB transcription factors

\section{Introduction}

Transcription factors (TFs) regulate various developmental and physiological processes in plants via binding to promoters of target genes to activate or suppress gene expression. The typical TFs commonly contain DNA-binding domains (DBDs), and can be classified into different families based on the sequence difference of DBDs [1]. Over 60 TF families with hundreds to thousands of members have been identified in higher plants, and only a few have been functionally analyzed [2]. The MYB transcription factor gene family is one of the largest TF families and is widely dispersed in plants. Members of the MYB gene family contain a N-terminal MYB DNA-binding domain, and the MYB domain frequently consists of one or more imperfect tandem repeats [3]. According to the number 
of MYB repeats, plant MYBs can be further classified into four major types (1R-MYB, R2R3-MYB, 3R-MYB and 4R-MYB); each MYB repeat contains about 50-55 amino acid residues and encodes three $\alpha$-helices [4]. Generally, the R2R3-MYB subfamily is the largest within the MYB family, and functions in diverse aspects of plant biology including primary and secondary metabolism, development and response to biotic and abiotic stresses [5].

In recent years, hundreds of plant genome sequences have been released in the "omics" era, and the R2R3-MYB gene family has been identified in various plants. A total of 126 R2R3-MYB members were identified in Arabidopsis, and 192 were found in the woody model plant Populus trichocarpa [6]. Analysis of the distribution of the R2R3-MYB gene family across 50 eukaryotes revealed that this gene family underwent a dramatic expansion in flowering plants [7].

Grafting has been applied in agriculture for more than 2000 years. Plant grafting, consisting of cutting and joining two plants growing together as one, is an ancient technology and widely used to increase production, modify branch architecture and fruit size, and improve stress tolerance. Today, grafting is still applied to propagate diverse fruit trees including pecans (Carya illinoinensis), pears (Pyrus bretschneideri), apples (Malus domestica) and grapevines (Vitis vinifera) [8]. As a successful grafting, graft union formation comprises callus formation and the creation of a functional vascular system [9]. The graft union formation in a grapevine was related to cell wall modification and secondary metabolism [10]. Most studies of grafting have focused on morphological and physiological observations; however, knowledge about the molecular mechanisms remains limited.

Callus formation and vascular reconstruction are both important for successful grafting, and MYB TFs related to these processes might function in plant grafting [11]. A MYB transcription factor, $A P L$, functions in phloem development and inhibits xylem differentiation [12]. Secondary cell walls are composed of lignin, cellulose, and hemicelluloses. In vascular plants, lignin is the second largest biopolymer, and plays a key role in offering a structural barrier to the cell wall. EgMYB1, an R2R3-MYB gene in eucalyptus, is preferentially expressed in differentiating xylem, and overexpression of this gene in Arabidopsis and poplar results in a decrease in lignin content [13]. AtMYB58 and AtMYB63 lead to the activation of lignin biosynthesis, and lignin biosynthetic genes are also induced in both overexpression lines [14]. AtMYB46 plays a key role in the regulation network of secondary wall biosynthesis [15].

Pecan [Carya illinoinensis (Wangenh.) K. Koch] is an important nut tree species which belongs to the genus Carya. It is native to the United States and Mexico. The seeds of pecans are a rich source of unsaturated fatty acids, proteins, B vitamins and abundant bioactive substances. Due to high nutritional value and significant human health benefits, pecan trees are widely planted and have an important economic value. The production of pecan nuts occurs mainly in Mexico and the United States. In the United States, pecan production exceeds 130,000 tons per year with a value over 600 million dollars [16]. Grafting, an asexual propagation technique, is widely used in the commercial pecan industry to reduce the duration of the juvenile phase and increase the nut production. Different pecan cultivars commonly have different graft success rates. The recent release of the pecan genome sequence allows the characterization of the $R 2 R 3-M Y B$ genes, and their expression levels during grafting based on the transcriptome data [16]. In this study, 153 R2R3-MYB family members were identified in the pecan and classified into different clades according to the phylogenetic analysis. The duplication events, expression patterns and co-expression networks were further analyzed to screen candidates functioned in the grafting process in the pecan. Overall, this study illustrated the genome-wide analysis of the R2R3-MYB gene family and identified potential candidates during the grafting process of the pecan.

\section{Materials and Methods}

\subsection{R2R3-MYB Identification in Five Different Plant Species}

To identify R2R3-MYB members from different plant genomes, all pecan (Carya illinoinensis), and Chinese hickory (Carya cathayensis) protein sequences were retrieved from the GigaScience database, 
GigaDB (http://gigadb.org/dataset/100571) [16]. The protein sequences of Arabidopsis (Arabidopsis thaliana), rice (Oryza sativa) and poplar (Populus trichocarpa) were obtained from Phytozome V12 database (https://phytozome.jgi.doe.gov/pz/portal.html). The hidden Markov model (HMM) profile of MYB DNA-binding-domain (PF00249) was downloaded from Pfam (http://pfam.xfam.org/) [17] and used as a query to investigate MYBs in different plants using HMMER version 3.1b2 software (www.hmmer.org) with an E-value cut-off of $<1.0 \times 10^{-5}$ [18]. The candidate R2R3-MYB proteins were further examined to confirm the presence of two MYB repeats using Pfam and SMART software (http://smart.embl-heidelberg.de/) [19].

\subsection{In Silico Analysis of Pecan R2R3-MYB Sequences}

Molecular weight (MW), isoelectric points (pIs), instability indexes and grand average of hydropathicity (GRAVY) of pecan R2R3-MYB proteins were calculated by ExPASy ProtParam server (http://web.expasy.org/protparam). The subcellular localization information of pecan R2R3-MYBs was predicted using CELLO v.2.5 (http://cello.life.nctu.edu.tw/) [20].

\subsection{Gene Structure and Conserved Motif Analysis}

Gene structure information of pecan R2R3-MYBs was retrieved by surveying the General Feature Format (GFF) file from GigaDB (http://gigadb.org/dataset/100571) [16]. The gene structural diagram of pecan R2R3-MYB was generated using TBtools software (https://github.com/CJ-Chen/TBtools) [21]. Conserved motifs of pecan R2R3-MYB protein sequences were identified by the MEME program (http://meme-suite.org/tools/meme) [22] with the search number of 30, and the optimum motif length between 6 and 200 residues [23]. To analyze the sequence features of the conserved MYB domain, the online WebLogo tool (http://weblogo.berkeley.edu/logo.cgi) was applied to generate the sequence logo after retrieving the MYB DNA-binding-domain sequences by Perl script [24].

\subsection{Sequence Alignment and Phylogenetic Tree Construction}

All R2R3-MYB protein sequences were aligned using MAFFT v7.397 software (https://mafft.cbrc. jp/alignment/software/) with G-INS-i strategy [25]. The phylogenetic tree was derived using FastTree v2.1.10 (http://www.microbesonline.org/fasttree/) with default parameters [26]. The resulting tree was visualized using MEGA 7 software [27].

\subsection{GO Functional Identification}

The pecan R2R3-MYB protein sequences were compared against the National Center for Biotechnology Information (NCBI) database using BLASTP. The Gene Ontology (GO) terms of functional annotation were analyzed using Blast2GO software (https://www.blast2go.com/) with default parameters [28], and annotations of GO terms were investigated using Gene Ontology Consortium (http://geneontology.org).

\subsection{Synteny Analysis}

For detecting syntenic blocks, the R2R3-MYB protein sequences from pecan were searched against themselves using the BLASTP tool NCBI-BLAST 2.7.1+ [29]. Then, MCScanX software was used to identify duplication events in the R2R3-MYB gene family in pecan [30].

\subsection{Estimation of the Ka/Ks Values}

Multiple sequence alignment of the full-length coding sequences (CDS) in the R2R3-MYB gene family in pecan were performed using ClustalW software version 2.1 [31]. MEGA 7 software was further used to calculate the nonsynonymous $(K a)$ and synonymous substitutions $(K s)$, and the ratio of $K a$ to $K s(K a / K s)$. Ks values are commonly used to determine the time since gene duplication, and the selection pressure of duplication event is determined by the $\mathrm{Ka} / \mathrm{Ks}$ ratio [32]. 


\subsection{Transcriptome Analysis}

Three biological replicates of samples from graft unions were collected at $0,8,15$ and $30 \mathrm{~d}$ after grafting, and used for transcriptome analysis. The transcript abundances of pecan $R 2 R 3-M Y B$ genes were calculated with FPKM (fragments per kilobase per million of reads mapped) values. The RNA-seq raw data can be accessed at the NCBI database (https://www.ncbi.nlm.nih.gov/) under the accession number PRJNA411951. Heatmaps of the pecan R2R3-MYB gene expression data were generated using the pheatmap package in R version 3.5.3 (https://www.r-project.org).

\subsection{Co-Expression Network Construction}

Pearson correlation coefficients (PCCs) of R2R3-MYB gene pairs were calculated using IBM SPSS Statistics 25 (SPSS Inc., Chicago, IL, USA) based on the transcriptome data. The gene pairs whose absolute value of PCCs greater than 0.9 at the desired significance level $(p$-value $<0.05)$ were used for network construction. The regulatory network was visualized and analyzed using Cytoscape v3.7.1 [33].

\subsection{Plant Materials and Sample Collection}

One-year-old pecan trees propagated by seeds were cultured and used as rootstocks, and the Pawnee cultivar acted as scion. Patch budding was applied in August for pecan grafting in a greenhouse at the campus of Nanjing Forestry University. On the basis of the morphological observation [9], samples from scion/rootstock close to the graft interface were harvested at 0, 8, 15 and $30 \mathrm{~d}$ after grafting. All samples were collected at about $5 \mathrm{pm}$, and further flash-frozen in liquid nitrogen, and stored at $-80{ }^{\circ} \mathrm{C}$ until RNA isolation. Three biological replicates were collected at each time point, and samples in each replicate were collected from three grafted plants.

\subsection{RNA Collection and Quantitative Real-Time PCR}

Total RNA was isolated using a Plant Total RNA Extraction Kit (BioTeke, Beijing, China) according to the manufacturer's instructions. DNase I (Qiagen, Hilden, Germany) was applied to reduce the genomic DNA contamination, and RNA concentration and quality were further detected using a NanoDrop 2000 spectrophotometer (Thermo Fisher Scientific, Wilmington, NC, USA). The first strand cDNAs were synthesized from $1 \mu \mathrm{g}$ of total RNA using Prime-Script RT Reagent Kit (TaKaRa, Dalian, China) following the manufacturer's instructions.

Quantitative real-time PCR (qRT-PCR) analysis was carried out on the 7500 Fast Real-Time PCR system (Applied Biosystems ${ }^{\mathrm{TM}}$, Foster city, CA, USA) with TB Green Premix Ex Taq II (TaKaRa, Shiga, Japan). Each reaction contained $10 \mu \mathrm{L}$ TB Green, $1 \mu \mathrm{L}$ cDNA, $1 \mu \mathrm{L}$ gene-specific primer, and additional nuclease-free water to a total volume of $20 \mu \mathrm{L}$. Specific PCR primers for pecan $R 2 R 3-M Y B$ genes were then designed using the IDT PrimerQuest (https://sg.idtdna.com/pages/tools/primerquest); specific primer sequences are listed in Table S1. CIL1155S0051, an actin gene used in previous studies, was selected as a reference gene to normalize the expression levels of target $R 2 R 3-M Y B$ genes [9]. The PCR parameters applied here were as follows: $95^{\circ} \mathrm{C}$ for $30 \mathrm{~s}$, followed by 40 cycles of $5 \mathrm{~s}$ at $95{ }^{\circ} \mathrm{C}$ and $15 \mathrm{~s}$ at $60^{\circ} \mathrm{C}$. Finally, melting curve analyses were performed to verify the specificity of primers. Relative expression levels of pecan $R 2 R 3-M Y B$ genes were determined using the $2^{-\Delta \Delta C t}$ method [23]. Values represent mean calculated from three biological replicates and three technological repeats.

\subsection{Cis-Regulatory Elements Analysis}

To investigate potential cis-regulatory elements in the promoters of pecan $R 2 R 3-M Y B$ genes, $2000 \mathrm{bp}$ regions upstream of the $R 2 R 3-M Y B$ genes were retrieved from the pecan genome sequences. Then, the cis-regulatory elements were predicted using PlantCARE software (http://bioinformatics.psb. ugent.be/webtools/plantcare/html/). The hormone response related elements in the $2000 \mathrm{bp}$ upstream regions of pecan $R 2 R 3-M Y B$ genes were selected and visualized by TBtools software [21]. 


\subsection{Statistical Analysis}

IBM SPSS Statistics 25 (SPSS Inc.) was employed for statistical analysis, and Duncan's multiple range test was conducted for statistical significance $(p<0.05)$.

\section{Results}

\subsection{Genome-Wide Identification of the R2R3-MYB Gene Family Members in Pecan}

The putative MYB transcription factors in the pecan genome were identified by HMMER and confirmed by the existence of MYB domain. A total of 328 MYB transcription factor candidates were identified that contained MYB or MYB-like repeats. After verifying the MYB domain by Pfam and SMART, 153 were identified as R2R3-MYB (2R-MYB) gene family members which contained two MYB repeats (Table S2). One 4R-MYB (CIL1098S0014), four 3R-MYB (CIL0922S0218, CIL1246S0130, CIL1439S0013, and CIL1218S0045), and 161 1R-MYBs were further identified in the pecan genome. Sequence analyses of the members of this gene family revealed that the gene length of R2R3-MYBs in pecan varied from 624 to 38,366 base pairs (bp), with proteins ranging in size from 161 to 1011 amino acids (Table S3). The relative molecular weights (MWs) of pecan R2R3-MYB proteins varied from 18,310.91 (CIL0946S0118) to 112,880.12 (CIL0057S0012) Dalton (Da), with isoelectric points (pIs) ranging from 4.74 (CIL0895S0131) to 9.57 (CIL1098S0008).

Subcellular localization information provided key cues to the potential function of protein. Subcellular localization prediction results showed that the majority of the R2R3-MYBs (149/153) localize to the nucleus. The instability indexes of pecan R2R3-MYB proteins varied from 27.71 (CIL1365S0032) to 68.77 (CIL1055S0121); most of these (147/153) were unstable. The grand average of hydropathicity (GRAVY) values indicated that the hydropathicity of the R2R3-MYB proteins in pecan was $<0$, suggesting that these proteins were hydrophilic. Detailed information of the R2R3-MYBs is provided in Table S3.

\subsection{Phylogenetic Analyses of R2R3-MYBs in Five Different Species}

A phylogenetic tree of pecan R2R3-MYBs with four additional important plant species was derived to investigate the phylogenetic relationships of R2R3-MYBs in different species. Four plant species were selected, namely, three dicotyledon plants (Arabidopsis, Populus trichocarpa, and Carya cathayensis) and a monocotyledon plant (Oryza sativa); P. trichocarpa (poplar) and C. cathayensis (Chinese hickory) are perennial woody plants. The pecan genome encoded more R2R3-MYB genes (153) than Arabidopsis (126) and rice (110), but less than those in poplar (200) and Chinese hickory (181). According to the phylogenetic analyses, in total, 770 R2R3-MYB proteins in the five plants were divided into 43 clades, namely, C1 to C40, and three additional woody plant preferential clades (W1 to W3) (Figure 1A). The phylogenetic tree showed that the majority of clades (32/43) contained family members from all five species, indicating that they might exist in their ancestral genome (Figure 1B). The remaining 11 clades were detected only in some of the five species including three woody plant preferential clades. Fifteen $R 2 R 3-M Y B$ genes in pecan belonged to the woody plant preferential clades. Furthermore, 16 and 14 woody plant preferential clade members existed in Chinese hickory and poplar, respectively. On the contrary, no woody plant preferential clade members were identified in Arabidopsis and rice, two herbaceous species. The detailed information of this phylogenetic tree is provided in Figure S1 and Table S3. 
A

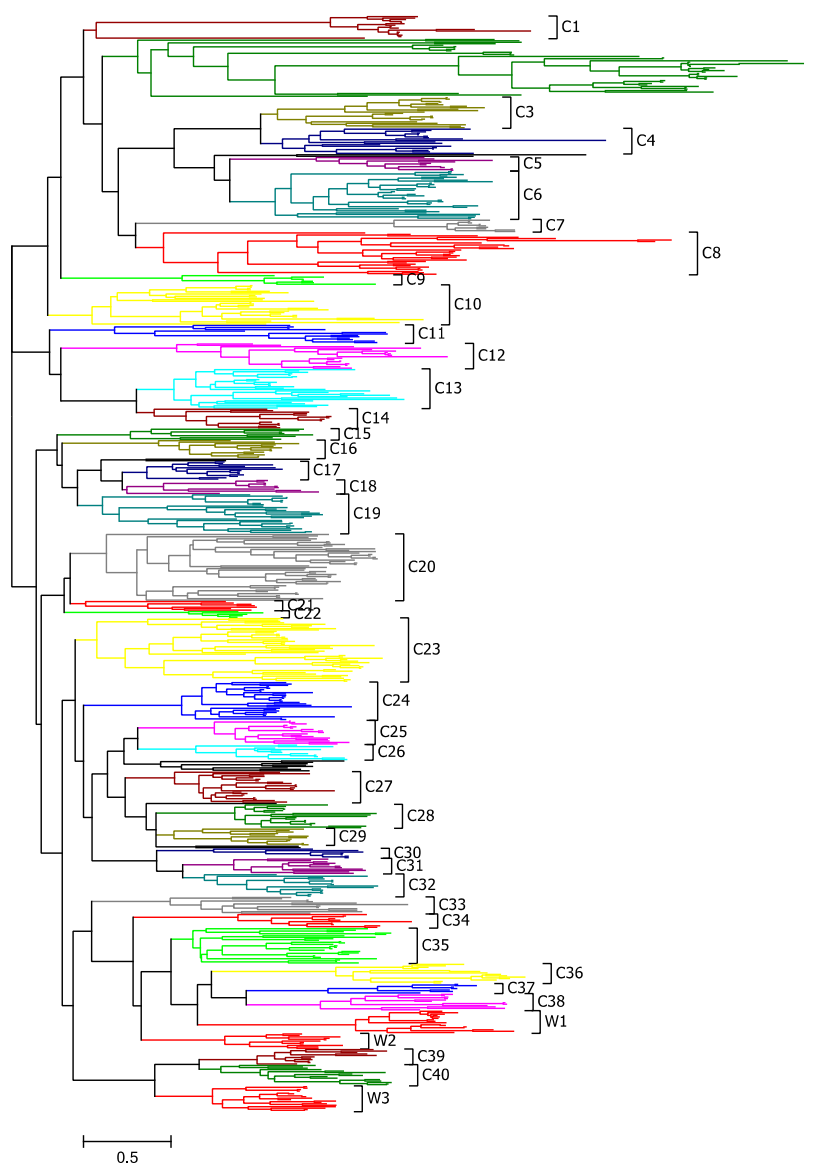

B

\begin{tabular}{|c|c|c|c|c|c|}
\hline Clade & Cil & $\mathrm{Cca}$ & At & Os & $\mathrm{Pt}$ \\
\hline C1 & 4 & 7 & 1 & 1 & 3 \\
\hline $\mathrm{C} 2$ & 9 & 12 & 0 & 7 & 13 \\
\hline C3 & 4 & 5 & 3 & 2 & 8 \\
\hline $\mathrm{C} 4$ & 3 & 3 & 5 & 2 & 5 \\
\hline $\mathrm{C} 5$ & 2 & 2 & 3 & 1 & 2 \\
\hline C6 & 8 & 8 & 4 & 5 & 9 \\
\hline C7 & 2 & 2 & 2 & 1 & 2 \\
\hline C8 & 6 & 6 & 7 & 5 & 6 \\
\hline C9 & 1 & 2 & 1 & 1 & 2 \\
\hline C10 & 5 & 6 & 7 & 5 & 5 \\
\hline C11 & 3 & 1 & 3 & 3 & 3 \\
\hline $\mathrm{C} 12$ & 2 & 4 & 3 & 3 & 6 \\
\hline $\mathrm{C} 13$ & 4 & 4 & 7 & 8 & 5 \\
\hline C14 & 3 & 4 & 2 & 1 & 4 \\
\hline C15 & 1 & 1 & 3 & 2 & 1 \\
\hline C16 & 3 & 3 & 2 & 1 & 4 \\
\hline C17 & 2 & 1 & 3 & 2 & 5 \\
\hline C18 & 2 & 2 & 1 & 3 & 2 \\
\hline $\mathrm{C} 19$ & 6 & 9 & 3 & 3 & 7 \\
\hline C20 & 10 & 12 & 6 & 9 & 10 \\
\hline C21 & 0 & 2 & 1 & 1 & 3 \\
\hline $\mathrm{C} 22$ & 1 & 0 & 1 & 1 & 2 \\
\hline $\mathrm{C} 23$ & 7 & 10 & 7 & 5 & 16 \\
\hline C24 & 6 & 5 & 5 & 7 & 4 \\
\hline C25 & 1 & 7 & 2 & 3 & 4 \\
\hline C26 & 3 & 1 & 1 & 2 & 4 \\
\hline C27 & 6 & 5 & 4 & 1 & 6 \\
\hline C28 & 3 & 4 & 3 & 3 & 4 \\
\hline C29 & 2 & 4 & 3 & 0 & 3 \\
\hline C30 & 2 & 2 & 1 & 0 & 2 \\
\hline C31 & 2 & 1 & 2 & 4 & 2 \\
\hline C32 & 4 & 4 & 3 & 1 & 4 \\
\hline $\mathrm{C} 33$ & 2 & 2 & 3 & 2 & 3 \\
\hline C34 & 1 & 1 & 1 & 1 & 6 \\
\hline C 35 & 6 & 11 & 1 & 2 & 5 \\
\hline C36 & 2 & 3 & 4 & 0 & 5 \\
\hline C37 & 2 & 2 & 1 & 0 & 2 \\
\hline C38 & 4 & 3 & 3 & 0 & 2 \\
\hline C39 & 2 & 1 & 3 & 2 & 3 \\
\hline C40 & 2 & 3 & 3 & 3 & 4 \\
\hline W1 & 6 & 6 & 0 & 0 & 4 \\
\hline W2 & 2 & 4 & 0 & 0 & 5 \\
\hline W3 & 7 & 6 & 0 & 0 & 5 \\
\hline
\end{tabular}

Figure 1. Phylogenetic analysis of R2R3-MYBs in five different plant species. (A) Phylogenetic tree of the R2R3-MYB proteins from pecan, Chinese hickory, Arabidopsis, rice and poplar. A total of 770 full-length R2R3-MYB proteins were retrieved from five plant species including Carya illinoinensis (Cil), Carya cathayensis (Cca), Arabidopsis thaliana (At), Oryza sativa (Os), and Populus trichocarpa (Pt). R2R3-MYB proteins from five species were divided into 43 clades (C1 to C40 and W1 to W3). (B) The number of R2R3-MYB proteins in each clade in different species. Detailed information of this phylogenetic tree is provided in Figure S1.

\subsection{Gene Structural and Conserved Motif Analyses of Pecan R2R3-MYBs}

To investigate the structural features of $R 2 R 3-M Y B$ genes in pecan, the exon/intron organization of these genes was analyzed. Exon numbers of pecan $R 2 R 3-M Y B$ genes ranged from 1 to 13 , and only ten were intron-less genes (Figure S2 and Table S3). Most R2R3-MYB genes possessed less than six exons, except CIL1535S0029 and CIL1277S0025, which contained 13 and 12 exons, respectively. The majority of pecan R2R3-MYB genes (103/153) contained three exons, 28 contained two exons, 7 contained four exons, and three contained five exons. In general, exon 1 and 2 encoded almost the entire R2 and R3 MYB repeats among the R2R3-MYB genes that contained three exons. Genes in the same clades commonly shared similar exon/intron patterns in pecan, and a similar phenomenon was also found in Arabidopsis and rice [34]; for example, all eight genes in C6 contained only one exon, and six genes in W1 had three exons (Figure S2).

Conserved motifs of all pecan R2R3-MYBs were also examined using the MEME program, and 30 motifs were detected; the related sequence information of different motifs are listed in Table S4. Based on the phylogenetic tree constructed using the 153 protein sequences, the pecan R2R3-MYB gene family was divided into various clades (Figure 2A). Generally, genes in the same clade shared similar motif organization patterns due to the high sequence similarity (Figure 2B), particularly the 
closely related R2R3-MYB proteins. For example, motif 21 only existed in R2R3-MYB proteins which were classified to C23, and motif 19 was only identified in six members in C24 (Figure S3). Almost all R2R3-MYBs in pecan contained motif 1, 2 and 3.

A

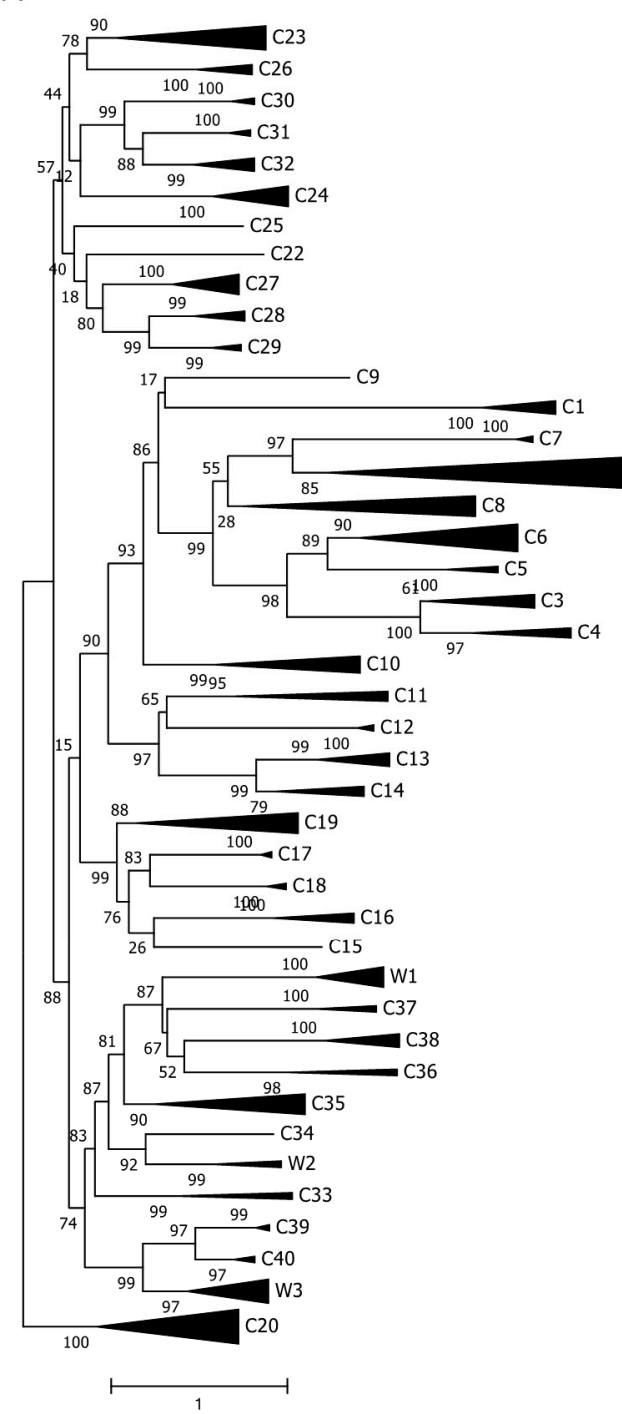

\section{B}

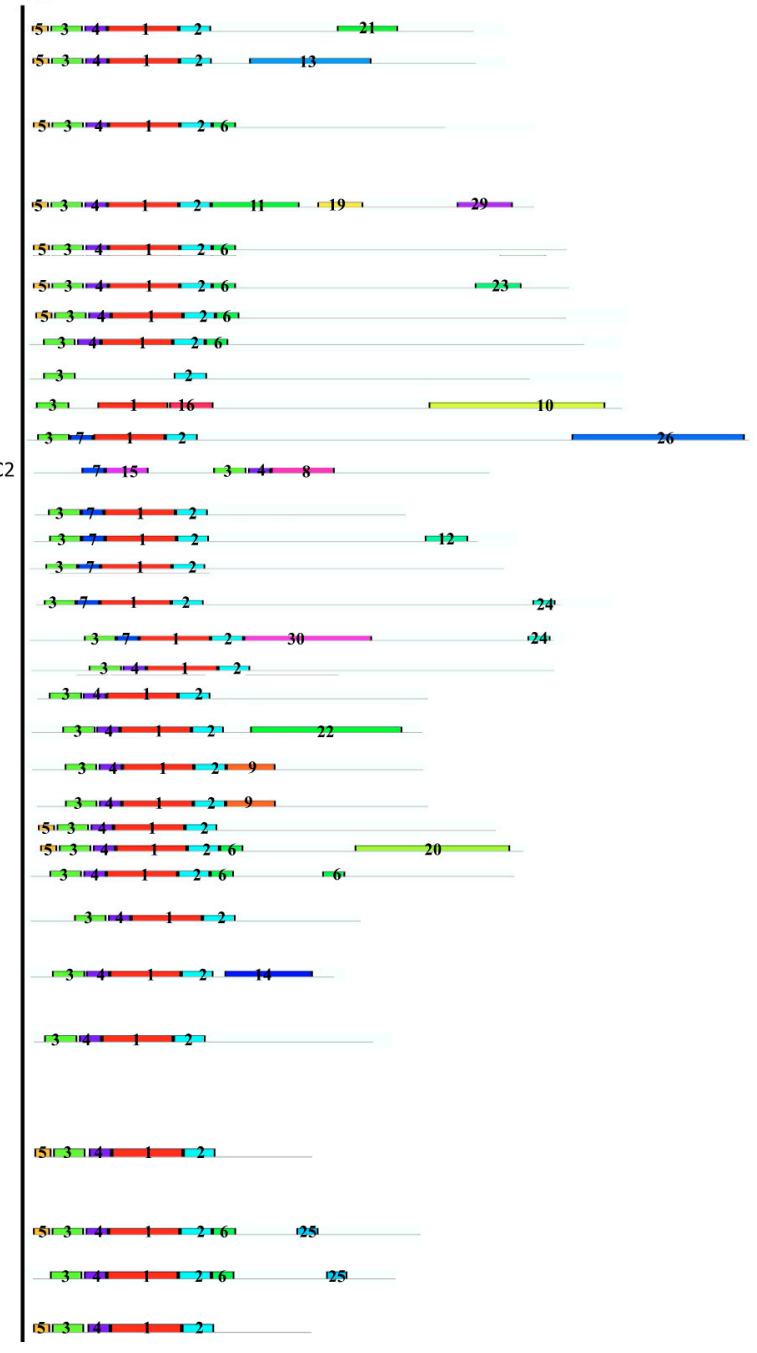

Figure 2. Phylogenetic relationship and representative conserved motif patterns in different pecan R2R3-MYB clades. Phylogenetic relationship of different clades (A), and typical motif patterns of R2R3-MYB members in different clades were identified (B). Detailed information of the motif pattern of the pecan R2R3-MYB gene family is provided in Figure S3, and sequence information of each motif is listed in Table S4.

R2R3-MYB proteins generally possessed two MYB repeats, named R2 and R3. To investigate the characteristics of MYB domain sequences and the degree of conservation of different residues, sequence logos of R2 and R3 repeats from pecan were created using WebLogo software (Figure 3). Three highly conserved tryptophan (Trp) residues were found in the R2 repeats of pecan R2R3-MYBs at positions 4, 27 and 48, while only two conserved Trp residues were identified at positions 24 and 44 of the $\mathrm{R} 3$ repeats (Figure 3 ). 
A
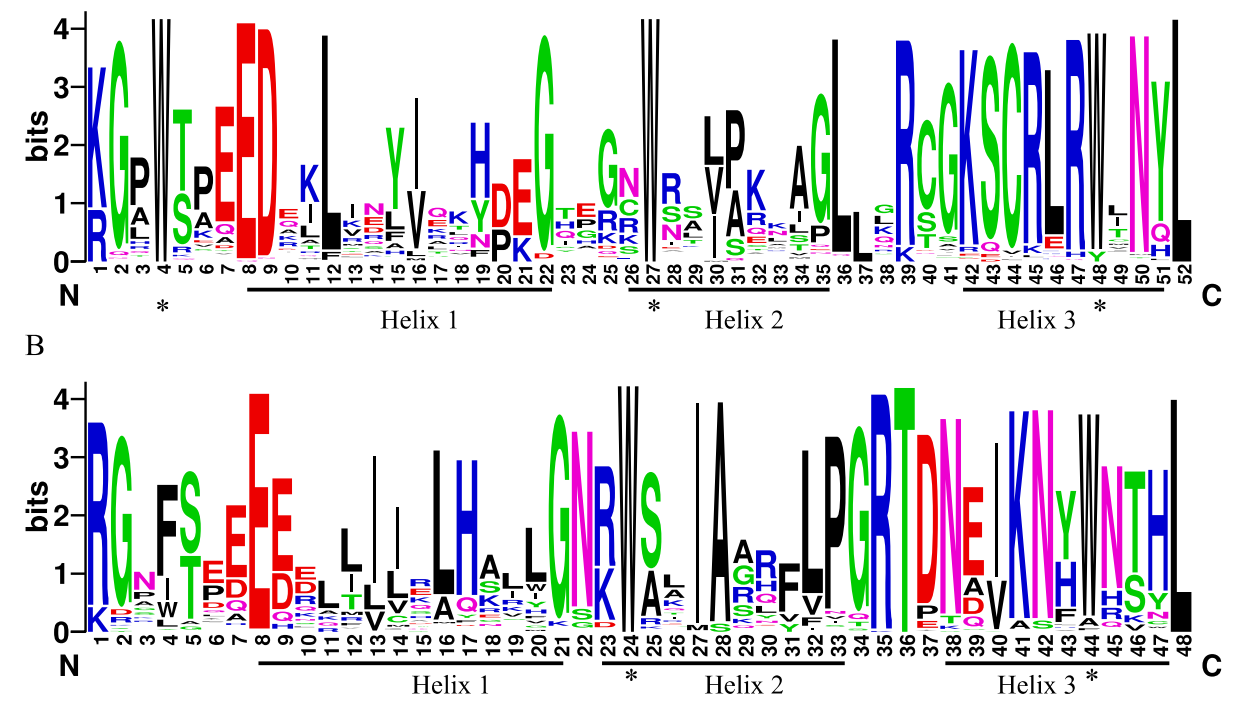

Figure 3. Highly conserved R2 and R3 MYB repeats across all R2R3-MYBs in pecan. Sequence logos of R2 (A), and R3 (B) repeats were generated by WebLogo based on the 153 pecan R2R3-MYB domain sequences. The height of each stack represents the conservation of the given position of MYB repeat sequences, and the height of different letters within each stack reveals the relative frequency of the amino acid. The asterisks ${ }^{*}$ ) represent the conserved tryptophan residues $(W)$ in the MYB repeats.

\subsection{Gene Ontology Functional Analysis of Pecan R2R3-MYBs}

Molecular function (MF), biological process (BP), and cellular component (CC) are three main GO categories, and are characteristics of genes that help to illuminate various functions of proteins [35]. GO annotations of 153 pecan R2R3-MYBs were further investigated using Blast2GO software. We found that the R2R3-MYBs were involved in all three GO categories; the largest portion (41.95\%) of GO terms were involved in the MF category (Figure $4 \mathrm{~A}$ ), 32.01\% were involved in the BP category and $26.04 \%$ were involved in the CC category. According to the MF results, all 153 genes were involved in DNA binding (GO:0003677), and 78 genes were related to DNA-binding transcription factor activity (GO:0003700), indicating the R2R3-MYBs bind to DNA to regulate the target genes (Figure 4B). Interestingly, 142 genes participated in the nucleus in CC (GO:0005634), suggesting that the majority are localized in the nucleus, which was consistent with the subcellular localization prediction results (Table S3). Moreover, 18 GO terms were found in the BP category, and the top three GO terms included cell differentiation (77 genes), biosynthetic process (40 genes), and cellular nitrogen compound metabolic process (39 genes), suggesting these pecan R2R3-MYBs are associated with various biological processes (Figure 4B).

\subsection{Ka, Ks Calculations and Selection Modes of Duplication Events}

Gene duplication contributes largely to the expansion of a gene family, and plays important roles in adaptation by obtaining novel gene functions [36]. MCScanX was used to detect the duplication events in the pecan R2R3-MYB gene family, and 52 duplicated gene pairs with 77 genes were found, indicating almost $50 \%$ of members were generated from gene duplication (Table S5).

Synonymous substitutions $(K s)$, such as a time indicator, were used to estimate the date of duplication events. The distribution of $K s$ values of duplication events in the pecan R2R3-MYB gene family revealed that the Ks varied from 0.33 to 4.81 and peaked in a range from 0.3 to 0.5 (Figure $5 \mathrm{~A}$ and Table S5). Surprisingly, the frequency of Ks values could be obviously divided into two parts, and the percentage of the frequency of $K s$ that ranged from 0.3 to 0.7 was $55.77 \%$. However, the remaining $44.23 \%$ of the frequency of $K s$ varied from 1.3 to 4.81 , revealing that recent duplications functioned in 
the expansion of the R2R3-MYB gene family in pecan (Table S5). The distributions of Ks in this gene family were further investigated, and the Ks values peaked at 2.2 to 2.4 (Figure $5 \mathrm{~B}$ ).

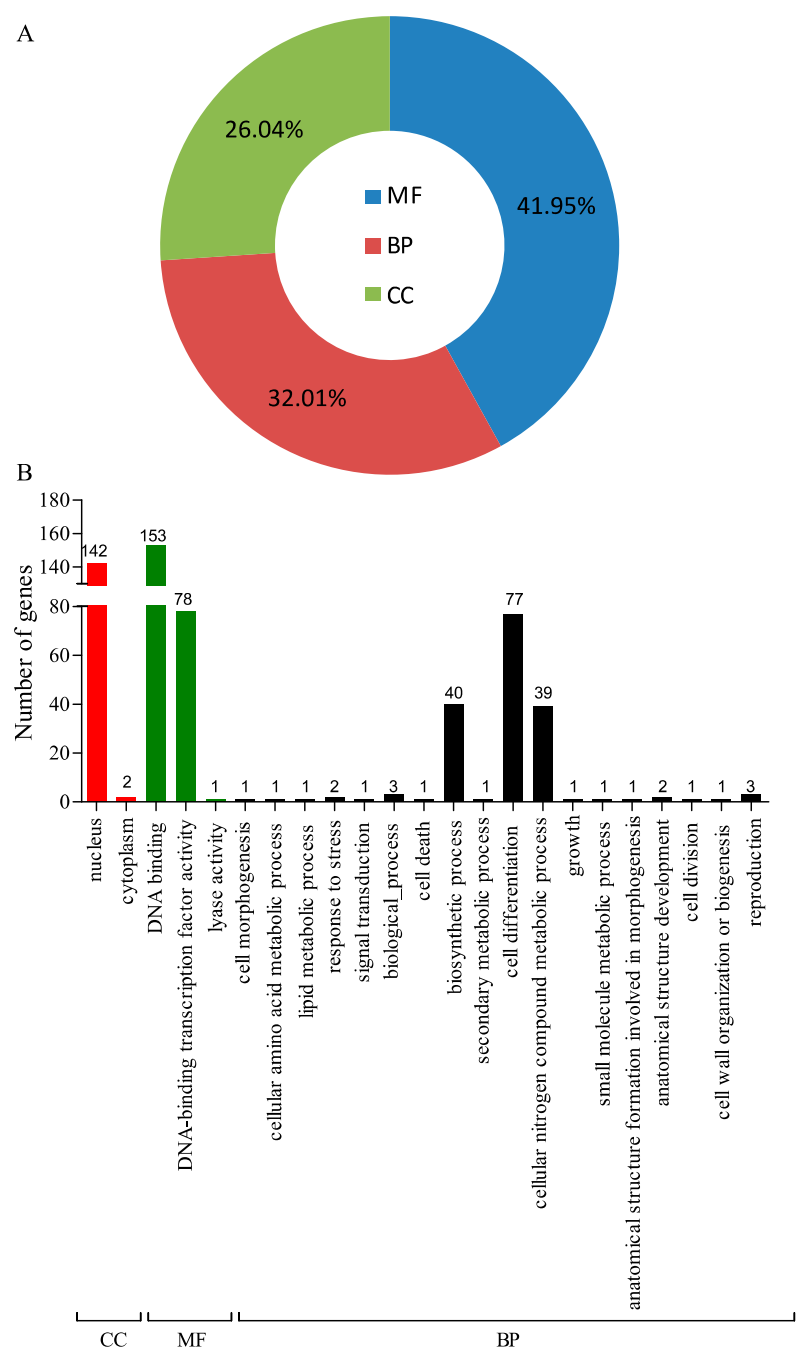

Figure 4. Gene Ontology (GO) analysis of $R 2 R 3-M Y B$ genes in pecan. CC: cellular component (red); MF: molecular function (green); BP: biological process (black). (A) Each slice in the pie chart represents the relative abundance of GO categories of 153 pecan R2R3-MYBs and their detailed classifications (B).

To examine the selection types of duplicated gene pairs in the pecan R2R3-MYB gene family, the non-synonymous $(\mathrm{Ka})$ and $K s$ substitution ratio $(\mathrm{Ka} / \mathrm{Ks})$ analysis of the duplication events was performed. A value of $K a / K s$ less than 1 indicates that the genes are under negative selection. $K a / K s>1$ implies that the genes have experienced positive selection, whereas $K a / K s=1$ means neutral selection. To our surprise, the Ka/Ks values of all the duplicated gene pairs were less than 1, suggesting that negative selection was the main influence on the expansion of pecan $R 2 R 3-M Y B$ genes, and played an essential role in the evolution of pecan R2R3-MYBs (Figure 5A). 


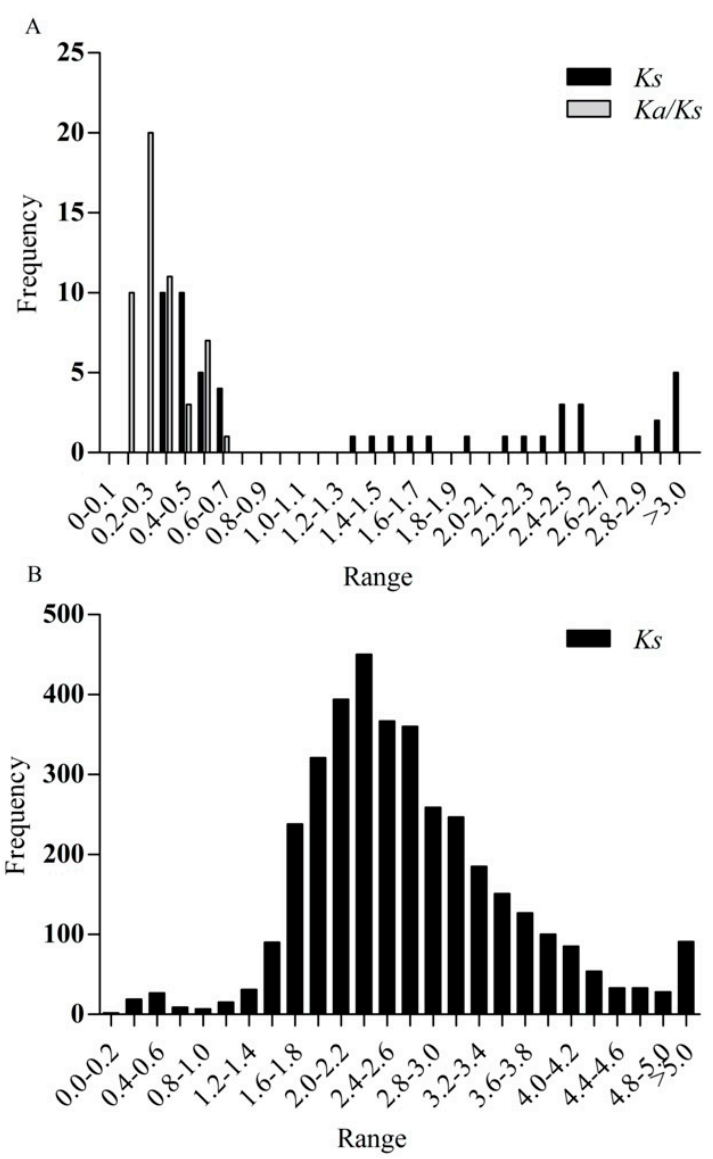

Figure 5. The frequency distribution of relative $K s$ and $K a / K s$ among duplication events (A) and relative Ks in the pecan R2R3-MYB gene family (B). The $X$-axis represents average Ks (black) or Ka/Ks (gray) per unit of 0.1 (A) or $0.2(\mathbf{B})$, and the $Y$-axis represents frequency.

\subsection{RNA-Seq and Co-Expression Analyses of Pecan R2R3-MYB Genes during Graft Union Formation}

Plant $R 2 R 3-M Y B$ genes regulate various biological processes including development and responses to biotic and abiotic stress [4]. To identify the potential roles of $R 2 R 3-M Y B$ genes in graft union formation in pecan, the transcriptome data during pecan graft union formation at four timepoints, namely, 0 , 8 (initial callus proliferation), 15 (callus formation and cambium establishment) and 30 (functional vascular bundles formation) days after grafting were further analyzed. In total, 149 available pecan $R 2 R 3-M Y B$ genes exhibited different expression patterns during graft union formation (Table S6). The heatmap with hierarchical clustering revealed that a portion of pecan R2R3-MYB genes in the same clade normally displayed similar expression patterns (Figure S4).

In total, 7305 differentially expressed genes (DEGs) with fold change $\geq 2$ and false discovery rate (FDR) $<0.01$ were identified according to the RNA-seq data; 4330 genes were significantly up-regulated (Figure S5A), and 2975 genes were significantly down-regulated during the graft union formation in pecan (Figure S5B). Among the DEGs, 64 genes were further identified as R2R3-MYB gene family members, with 47 genes up-regulated and 17 down-regulated (Figure 6). The 64 R2R3-MYB genes displayed different expression patterns, indicating that they participated in various biological processes. For example, five R2R3-MYB genes in C6 (CIL1107S0014, CIL1023S0011, CIL1207S0053, CIL0925S0130, and CIL1463S0018) all showed high expression levels at $15 \mathrm{~d}$ after grafting, suggesting they might be involved in callus formation and cambium establishment. 


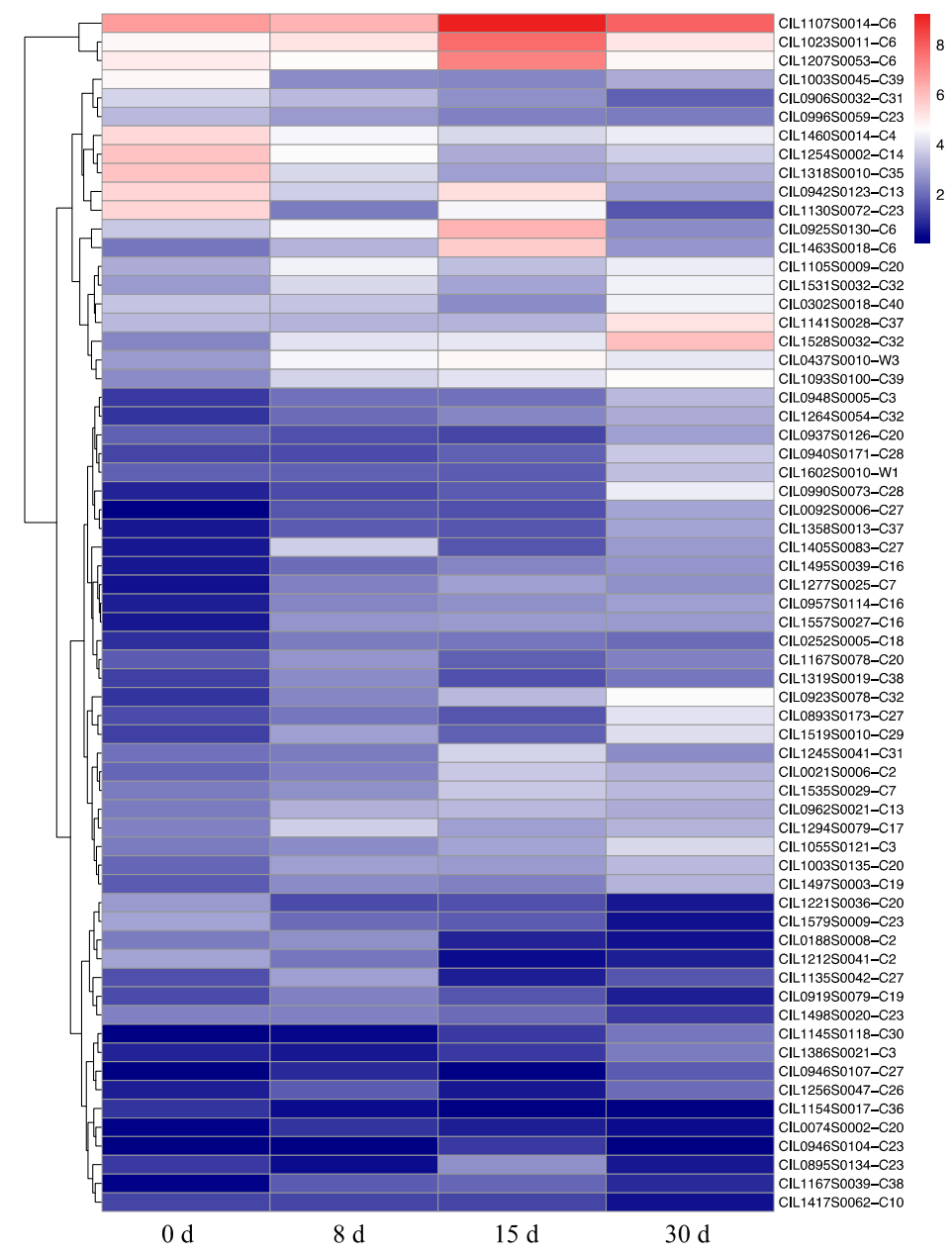

Figure 6. A heatmap of the expression levels of 64 differentially expressed $R 2 R 3-M Y B$ genes after grafting in pecan. The heatmap denoting the $\log _{2}(\mathrm{FPKM}+1)$ of target genes from RNA-seq data was derived using the pheatmap package with hierarchical clustering in $\mathrm{R}$ language. The color scale indicates gene expression levels, with purple representing low expression levels and red representing high levels.

Co-expression networks were further constructed to detect the mutual relationships between the 64 differentially expressed $R 2 R 3-M Y B$ genes based on the Pearson correlation coefficients (PCCs) of the expression data from RNA-seq. The co-regulatory networks contained 57 nodes (genes) and 219 edges (co-expression events), including one main network and two subnetworks (Figure 7). The main network contained 47 nodes, 15 of which had more than 10 edges, indicating these genes may play key roles in the graft union formation process. Among the 15 genes, CIL1528S0032 had the maximum number of edges (17). Intriguingly, the 15 genes were from nine clades, and most of these (10/15) were generated from gene duplication (Table S5). The other two subnetworks had two and eight nodes, with the number of edges varying from one to seven. In the co-expression networks, 172 of 219 co-expression events contained significantly positive correlations, and the remaining 47 events had significantly negative correlations. Interestingly, among the 15 genes that contained over ten edges, 14 were significantly up-regulated at different timepoints, with the exception of CIL0996S0059, which was significantly down-regulated at 30 days after grafting. 


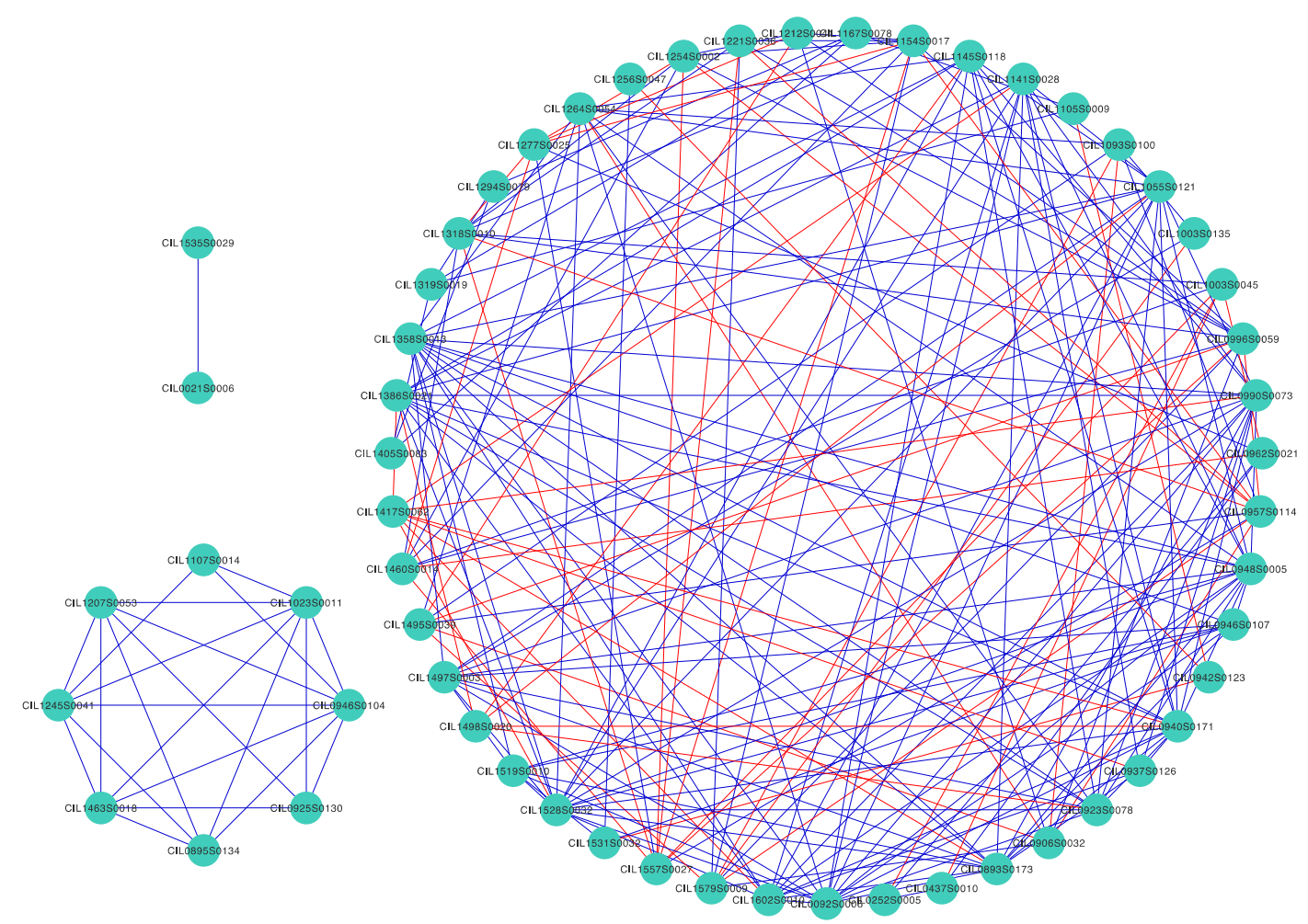

Figure 7. Co-expression networks of the 64 differentially expressed $R 2 R 3-M Y B$ genes in pecan after grafting. The blue line color represents positive correlations, and red represents negative correlations. The co-expression networks were visualized using Cytoscape software.

\section{7. qRT-PCR Quantification of Pecan R2R3-MYB Gene Expression Levels after Grafting}

Based on the co-expression networks (Figure 7), 12 pecan R2R3-MYB genes that co-expressed with more than 10 nodes were selected and further analyzed using quantitative real-time PCR (qRT-PCR). Surprisingly, the qRT-PCR results revealed that most genes displayed different expression patterns between scion and rootstock samples close to the graft interface (Figure 8). For example, CIL0893S0173 was significantly up-regulated in rootstock samples close to the graft interface, however, it was down-regulated in scion samples. In rootstock samples, ten of the 12 genes were up-regulated; only CIL1141S0028 was slightly down-regulated at $30 \mathrm{~d}$ after grafting, and CIL1602S0010 did not exhibit significant expression changes at each of the four timepoints. However, only three genes, namely, CIL0092S0006, CIL0990S0073 and CIL1141S0028, were up-regulated in rootstock samples, and the other nine genes were down-regulated. The hormone response-related cis-elements of 12 R2R3-MYB genes were investigated, and multiple hormone response-related cis-elements were found, including ABRE, CGTCA-motif, P-box, TATC-box, TCA-element and TGACG-motif, suggesting these genes may be involved in the hormone signaling pathway (Figure S6). 


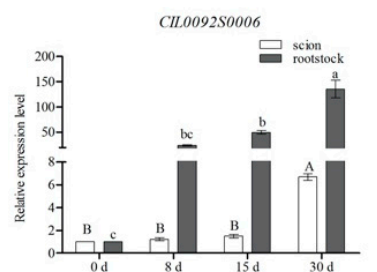

CIL105550121

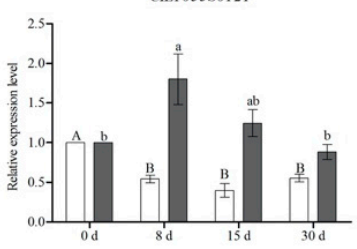

CLL13865002I
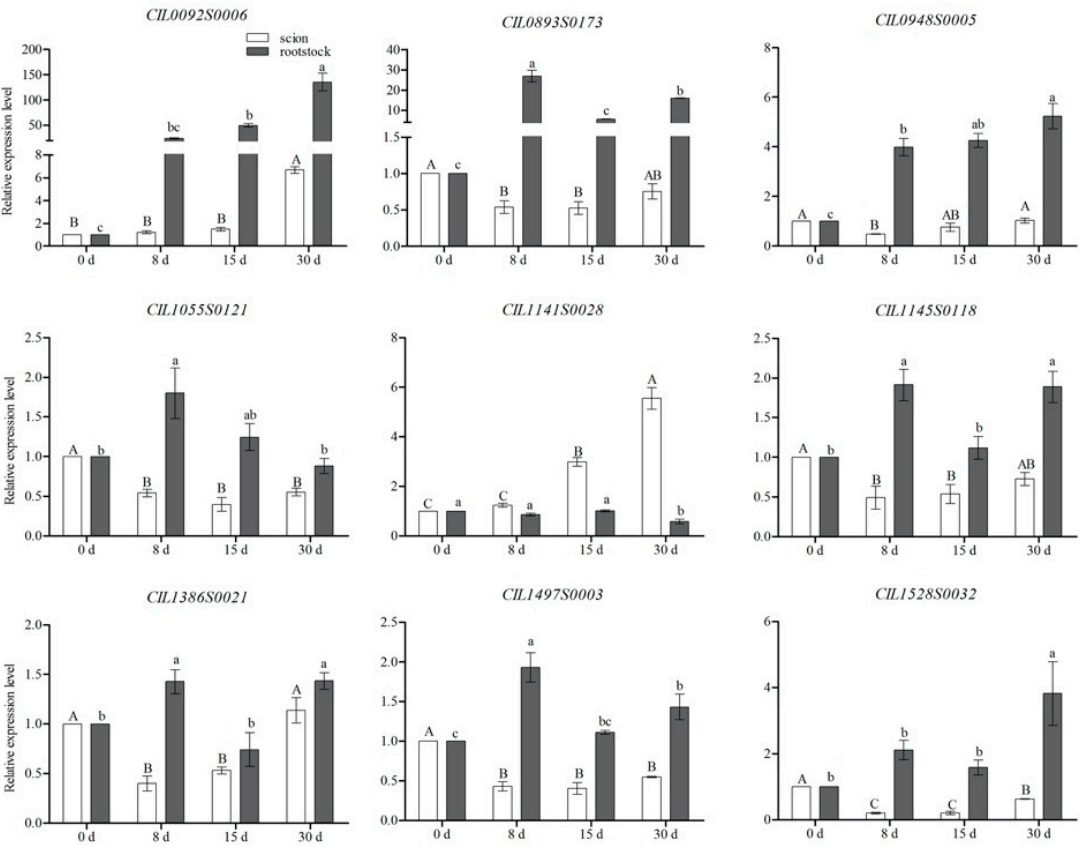

CLI 149750003

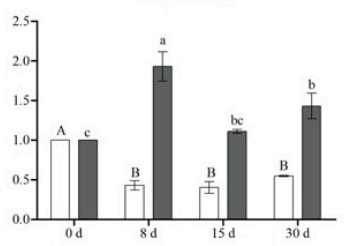

CLL1145S0118

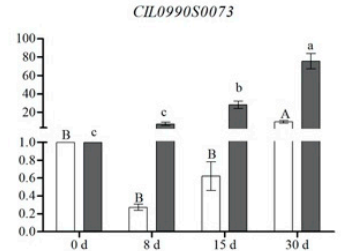

CLL135850013

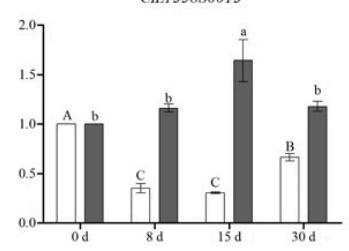

CLL1528S0032
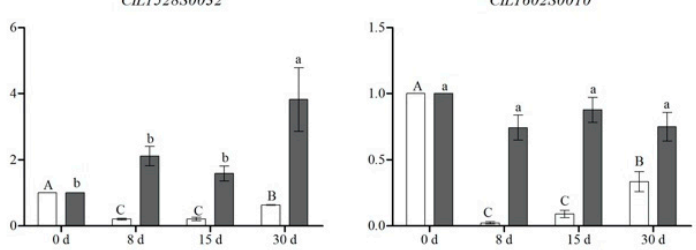

Figure 8. Quantification of expression levels of pecan R2R3-MYBs after grafting using qRT-PCR. One-year-old pecan trees were cultured as rootstocks, and Pawnee cultivar was used as scion. After grafting, samples from rootstock and scion close to the graft interface were collected at 0 (control), 8, 15 and $30 \mathrm{~d}$. The actin gene (CIL1155S0051) was applied as a reference gene. Values are means \pm Standard Error (SE) of three biological replicates, and different letters reveal significant difference $(p<0.05)$ according to Duncan's test.

CIL0092S0006 and CIL0990S0073 showed similar expression patterns in scion and rootstock. Surprisingly, the two genes were both induced gradually and displayed the highest expression levels at 30 days after grafting in rootstock samples. CIL0092S0006 was found to be up-regulated by more than 135 folds at $30 \mathrm{~d}$ compared with control $(0 \mathrm{~d})$, and CIL0990S0073 increased by over 75 folds, suggesting the two genes might play important roles during the grafting process, especially in vasculature formation.

\section{Discussion}

\subsection{Pecan Genome Possessed a Large R2R3-MYB Gene Family}

R2R3-MYB TFs function in various biological processes in plants including growth, development and response to stress stimuli, via binding to the promoter of target genes to activate or suppress gene expression [37]. The R2R3-MYB genes ubiquitously exist in all eukaryotes, and comprise a large transcription factor family in land plants, in contrast with other eukaryotes such as red and green algae [7]. Based on the release of the pecan genome sequence, 153 pecan $R 2 R 3-M Y B$ genes were identified, accounting for $\sim 0.49 \%(153 / 31,075)$ of the number of predicted genes in the pecan genome (Table S2) [16]. The size of the pecan R2R3-MYB gene family was larger than that in Arabidopsis (126) and rice (110), but smaller than that in some other plants, including Chinese hickory (181), poplar (200), cotton (205), apple (219) and sweet osmanthus (243) (Figure 1) [38-40]. According to an investigation of the R2R3-MYB gene family from fifty major lineages of eukaryotes, this gene family was proven to have experienced a substantial expansion in seed plants [7]. The R2R3-MYB gene family from five species was further classified into 43 clades, including three woody plant preferential (W) clades that only possessed members in three woody plants (Figure 1). Similarly, 141 Eucalyptus grandis R2R3-MYB genes were classified into 42 clades, and 15 members from five $W$ clades, indicating that diversification of specific functions of these genes occurred in woody plants [5]. 
The exon/intron structural pattern analysis showed approximately two-thirds of pecan $R 2 R 3-M Y B$ genes contained three exons and two introns (Figure S2); about $65 \%$ of pineapple R2R3-MYB genes possess similar exon/intron structures [41]. This phenomenon also occurs in the Eucalyptus grandis R2R3-MYB family, and exons 1 and 2 encoded almost the whole MYB domain [5]. R2R3-MYB proteins were characterized by two highly conserved MYB repeats, and each repeat contained three helices (Figure 3); the second and third helices generally formed a helix-turn-helix structure (Dubos et al., 2010). The sequence logos of $R 2$ and R 3 MYB repeats revealed that three highly conserved tryptophan (Trp) residues existed in R2; however, only two conserved Trp residues existed in R3 (Figure 3). The first Trp in R3 was mostly replaced by phenylalanine (F); this phenomenon was also observed in soybean and cotton [38,42]. These tryptophan residues were previously identified as a landmark of the MYB repeats. Members in each clade showed similar exon/intron and motif arrangements, indicating common evolutionary history within the same clade [39].

\subsection{Gene Duplication Contributed to the Expansion of Pecan R2R3-MYB Gene Family}

Gene duplication, as a type of genomic change, contributes to evolutionary novelties and provides new genes. In plant genomes, $65 \%$ of genes generally possess a duplicate copy [36]. The dramatic expansion of the R2R3 gene family in seed plants has been mainly attributed to gene duplication [7]. In the pecan R2R3-MYB gene family, about half of the members, with 52 duplication events, were generated from gene duplication, while 10,530 paralogous gene pairs were involved in pecan genome (Table S5) [16].

Positive selection collects new advantageous mutations and spreads throughout the population, however, negative selection is the process in which deleterious mutations are removed [43]. Ka/Ks ratios of all of the duplication events in the pecan R2R3-MYB gene family were less than 1, indicating that negative selection was the main influence on the expansion of the pecan R2R3-MYB gene family (Figure 5). Duplicated R2R3-MYB gene pairs in pineapple and sweet osmanthus were also under negative selection [40,41]. Whole genome duplication, tandem duplication and segmental duplication play critical roles in the expansion of gene families [44]. In the plant R2R3-MYB gene family, high segmental and low tandem duplication events have been reported in previous studies [41,42]. The distribution of $K s$ values in the duplication events revealed $55.77 \%$ of gene duplications in the pecan R2R3-MYB gene family occurred more recently, and the remaining $44.23 \%$ occurred during relatively distant periods (Figure 5).

\subsection{Expression Patterns of Pecan R2R3-MYB Genes during Graft Union Formation}

Grafting is a common horticulture technique. Two key processes for a successful graft include callus tissue formation and the connection of vascular tissues between scion and rootstock, while the molecular mechanisms are largely unknown [45]. According to the transcriptome profiles of the graft healing process of Litchi chinensis, $485 \mathrm{TFs}$ were identified as differentially expressed genes including 34 MYBs [46]. A large number of $R 2 R 3-M Y B$ genes have not been functionally characterized, especially in perennial woody plants, due to limited genome information. Because expression patterns might be associated with gene functions, we further analyzed the transcriptome data of pecan graft union formation processes after grafting. Pecan $R 2 R 3-M Y B$ genes revealed different expression patterns, and approximately one-third pecan $R 2 R 3-M Y B$ genes displayed low expression levels (Figure S4). A total of $41.83 \%$ (64/153) of pecan $R 2 R 3-M Y B$ genes were differentially expressed (Fold Change $\geq 2$, FDR $<0.01$ ), indicating that pecan $R 2 R 3-M Y B$ genes play important roles in the graft union formation process (Figure 6). The majority of genes in $\mathrm{C} 6$ showed high expression levels in $15 \mathrm{~d}$, indicating these genes might function in callus formation and cambium establishment, and genes in C6 expanded in woody plants specifically (Figure 1). Interestingly, AtMYB77 in C6 was involved in auxin signal transduction via its interaction with auxin response factors, suggesting these genes might be involved in the auxin signal transduction pathway [47]. Co-expression networks were further constructed and investigated to find the central nodes among these differentially expressed R2R3-MYB genes (Figure 7). 
A few $R 2 R 3-M Y B$ genes have been functionally analyzed, and a portion of these was found to be woody growth related. Lignin is one of most abundant biopolymers, and in wood, over $15 \%$ of the dry weight is lignin [46]. R2R3-MYBs have been proven to participate in lignin biosynthesis and secondary cell wall formation [5]. A eucalyptus $R 2 R 3-M Y B$ gene, EgMYB1, is involved in negatively regulating secondary cell wall formation, and overexpression of EgMYB1 in poplar reduces expression levels of lignin biosynthesis genes [13]. PtMYB4 is expressed in pine cells undergoing lignification, and overexpression of PtMYB4 in tobacco shows that lignin deposition has increased [48]. Populus R2R3-MYB genes also participate in the biosynthesis of specialized branches of the metabolism, including phenylpropanoid biosynthesis, a pathway that is related with phenolic compound biosynthesis [6]. Overexpression of SiMYB56 in rice increases the lignin biosynthesis-related gene expression levels and improves drought tolerance in transgenic rice plants [49].

According to the qRT-PCR results (Figure 8), we found CIL0092S0006 and CIL0990S0073 were significantly up-regulated during the pecan grafting process in both scion and rootstock samples. Potri.011G041600, a poplar R2R3-MYB gene that was in the same clade as CIL0092S0006 (Figure S1), was proven to be highly connected with lignin biosynthesis [50]. At5G10280 (AtMYB92), also in C28 with CIL0990S0073, acts as a candidate regulator of lignin deposition [51]. Although functional analyses of pecan $R 2 R 3-M Y B$ genes were limited, understanding the gene expression patterns might help us to understand the molecular mechanisms in graft union formation and find key candidate genes. Members of $A u x / I A A$ in Chinese hickory were identified and their expression profiles during the graft processing were analyzed to illuminate the roles of $A u x / I A A$ genes involved in graft union formation [52]. Based on the high sequence similarity of R2R3-MYBs in different perennial woody plants, it seems that this methodology could be extrapolated to other important forest trees including walnut and Chinese hickory to enhance the grafting success rate and reduce the duration of the juvenile phase.

\section{Conclusions}

Based on a comprehensive study, 153 R2R3-MYB genes were identified in the pecan genome and classified into different clades based on phylogenetic analyses. Gene duplication contributed largely to the expansion of the pecan R2R3-MYB gene family, and 52 duplication events were found. In addition, 77 pecan $R 2 R 3-M Y B$ genes generated from gene duplication with $\mathrm{Ka} / \mathrm{Ks}<1$ indicated that they had undergone negative selection. On the basis of the RNA-seq and co-expression network results, 15 differentially expressed $R 2 R 3-M Y B$ genes with high co-expression relationships might play key roles in graft union formation in pecan, and most of these were generated from gene duplication. According to the qRT-PCR results, CIL0092S0006 and CIL0990S0073 were both significantly up-regulated in scion and rootstock samples. These findings may help select candidate genes and contribute to further analysis on the functional identification of pecan R2R3-MYBs during the graft union formation.

Supplementary Materials: The following are available online at http://www.mdpi.com/1999-4907/11/9/917/s1, Figure S1: Phylogenetic tree representing relationships of the R2R3-MYB proteins from pecan, Chinese hickory, Arabidopsis, rice and poplar. Figure S2: Exon/intron structural patterns of R2R3-MYBs in pecan. Figure S3: Motif patterns of pecan R2R3-MYB proteins. Figure S4: A heatmap of the expression patterns of 149 pecan $R 2 R 3-M Y B$ genes during graft union formation. Figure S5: Venn diagram of differentially expressed in pecan after 8, 15 and 30 days of grafting compared with 0 days (control). Figure S6: Putative cis-elements in the promoters of pecan R2R3-MYB genes. Table S1: Specific primers of R2R3-MYB genes for qRT-PCR analysis. Table S2: MYB domain annotation of 153 pecan R2R3-MYBs. Table S3: List of 153 R2R3-MYB genes identified in pecan and their related information. Table S4: Motif sequences of pecan R2R3-MYBs. Table S5: Duplication events and related $\mathrm{Ka} / \mathrm{Ks}$ values of pecan R2R3-MYBs. Table S6: FPKM expression values of $153 \mathrm{R} 2 \mathrm{R} 3-\mathrm{MY} B$ genes during graft union formation.

Author Contributions: K.Z. and F.P. conceived and designed this research. K.Z. and F.P. wrote the manuscript. K.Z., P.F., F.L. and Z.M. performed the experiments. K.Z., P.T. and G.F. performed the data analysis. All authors modified the manuscript and approved the final MS. All authors have read and agreed to the published version of the manuscript. 
Funding: This research was mainly supported by a grant from National Natural Science Foundation of China (31870672) and the National Key R\&D Program of China (2018YFD1000604), and partially supported by a grant of the Natural Science Foundation of Jiangsu Province (BK20190749) and the NFU Scientific Research Startup Fund (163010226).

Conflicts of Interest: The authors declare no conflict of interest.

\section{References}

1. Riechmann, J.L.; Heard, J.; Martin, G.; Reuber, L.; Jiang, C.; Keddie, J.; Adam, L.; Pineda, O.; Ratcliffe, O.J.; Samaha, R.R.; et al. Arabidopsis transcription factors: Genome-wide comparative analysis among eukaryotes. Science 2000, 290, 2105-2110. [CrossRef] [PubMed]

2. Liu, J.; Chen, N.; Chen, F.; Cai, B.; Dal Santo, S.; Tornielli, G.; Pezzotti, M.; Cheng, Z.-M. Genome-wide analysis and expression profile of the bZIP transcription factor gene family in grapevine (Vitis vinifera). BMC Genom. 2014, 15, 281. [CrossRef] [PubMed]

3. Jin, H.; Martin, C. Multifunctionality and diversity within the plant MYB-gene family. Plant Mol. Biol. 1999, 41, 577-585. [CrossRef]

4. Millard, P.S.; Kragelund, B.B.; Burow, M. R2R3 MYB Transcription Factors-Functions outside the DNA-Binding Domain. Trends Plant Sci. 2019, 24, 934-946. [CrossRef] [PubMed]

5. Soler, M.; Camargo, E.L.O.; Carocha, V.; Cassan-Wang, H.; San Clemente, H.; Savelli, B.; Hefer, C.A.; Paiva, J.A.P.; Myburg, A.A.; Grima-Pettenati, J. The Eucalyptus grandis R2R3-MYB transcription factor family: Evidence for woody growth-related evolution and function. New Phytol. 2015, 206, 1364-1377. [CrossRef] [PubMed]

6. Wilkins, O.; Nahal, H.; Foong, J.; Provart, N.J.; Campbell, M.M. Expansion and diversification of the Populus R2R3-MYB family of transcription factors. Plant Physiol. 2009, 149, 981-993. [CrossRef] [PubMed]

7. Du, H.; Liang, Z.; Zhao, S.; Nan, M.-G.; Tran, L.-S.P.; Lu, K.; Huang, Y.-B.; Li, J.-N. The evolutionary history of R2R3-MYB proteins across 50 eukaryotes: New insights into subfamily classification and expansion. Sci. Rep. 2015, 5, 11037. [CrossRef]

8. Tsutsui, H.; Notaguchi, M. The use of grafting to study systemic signaling in plants. Plant Cell Physiol. 2017, 58, 1291-1301. [CrossRef]

9. Mo, Z.; Feng, G.; Su, W.; Liu, Z.; Peng, F. Transcriptomic analysis provides insights into grafting union development in pecan (Carya illinoinensis). Genes 2018, 9, 71. [CrossRef]

10. Cookson, S.J.; Clemente Moreno, M.J.; Hevin, C.; Nyamba Mendome, L.Z.; Delrot, S.; Trossat-Magnin, C.; Ollat, N. Graft union formation in grapevine induces transcriptional changes related to cell wall modification, wounding, hormone signalling, and secondary metabolism. J. Exp. Bot. 2013, 64, 2997-3008. [CrossRef]

11. Wang, J.; Jiang, L.; Wu, R. Plant grafting: How genetic exchange promotes vascular reconnection. New Phytol. 2017, 214, 56-65. [CrossRef] [PubMed]

12. Bonke, M.; Thitamadee, S.; Mähönen, A.P.; Hauser, M.-T.; Helariutta, Y. APL regulates vascular tissue identity in Arabidopsis. Nature 2003, 426, 181-186. [CrossRef] [PubMed]

13. Legay, S.; Sivadon, P.; Blervacq, A.-S.; Pavy, N.; Baghdady, A.; Tremblay, L.; Levasseur, C.; Ladouce, N.; Lapierre, C.; Séguin, A.; et al. EgMYB1, an R2R3 MYB transcription factor from eucalyptus negatively regulates secondary cell wall formation in Arabidopsis and poplar. New Phytol. 2010, 188, 774-786. [CrossRef] [PubMed]

14. Zhou, J.; Lee, C.; Zhong, R.; Ye, Z.-H. MYB58 and MYB63 are transcriptional activators of the lignin biosynthetic pathway during secondary cell wall formation in Arabidopsis. Plant Cell 2009, 21, $248-266$. [CrossRef] [PubMed]

15. Zhong, R.; Richardson, E.A.; Ye, Z.-H. The MYB46 transcription factor is a direct target of SND1 and regulates secondary wall biosynthesis in Arabidopsis. Plant Cell 2007, 19, 2776-2792. [CrossRef]

16. Huang, Y.; Xiao, L.; Zhang, Z.; Zhang, R.; Wang, Z.; Huang, C.; Huang, R.; Luan, Y.; Fan, T.; Wang, J.; et al. The genomes of pecan and Chinese hickory provide insights into Carya evolution and nut nutrition. GigaScience 2019, 8, giz036. [CrossRef]

17. El-Gebali, S.; Mistry, J.; Bateman, A.; Eddy, S.R.; Luciani, A.; Potter, S.C.; Qureshi, M.; Richardson, L.J.; Salazar, G.A.; Smart, A.; et al. The Pfam protein families database in 2019. Nucleic Acids Res. 2019, 47, D427-D432. [CrossRef] 
18. Eddy, S.R. Profile hidden Markov models. Bioinformatics 1998, 14, 755-763. [CrossRef]

19. Letunic, I.; Bork, P. 20 years of the SMART protein domain annotation resource. Nucleic Acids Res. 2018, 46, D493-D496. [CrossRef]

20. Yu, C.-S.; Chen, Y.-C.; Lu, C.-H.; Hwang, J.-K. Prediction of protein subcellular localization. Proteins 2006, 64, 643-651. [CrossRef]

21. Chen, C.; Chen, H.; Zhang, Y.; Thomas, H.R.; Frank, M.H.; He, Y.; Xia, R. TBtools-an integrative toolkit developed for interactive analyses of big biological data. Mol. Plant 2020. [CrossRef] [PubMed]

22. Bailey, T.L.; Johnson, J.; Grant, C.E.; Noble, W.S. The MEME Suite. Nucleic Acids Res. 2015, 43, W39-W49. [CrossRef] [PubMed]

23. Zhu, K.; Chen, F.; Liu, J.; Chen, X.; Hewezi, T.; Cheng, Z.-M. Evolution of an intron-poor cluster of the CIPK gene family and expression in response to drought stress in soybean. Sci. Rep. 2016, 6, 28225. [CrossRef] [PubMed]

24. Crooks, G.E. WebLogo: A Sequence Logo Generator. Genome Res. 2004, 14, 1188-1190. [CrossRef] [PubMed]

25. Katoh, K.; Rozewicki, J.; Yamada, K.D. MAFFT online service: Multiple sequence alignment, interactive sequence choice and visualization. Brief. Bioinform. 2019, 20, 1160-1166. [CrossRef]

26. Price, M.N.; Dehal, P.S.; Arkin, A.P. FastTree 2-approximately Maximum-Likelihood trees for large alignments. PLoS ONE 2010, 5, e9490. [CrossRef]

27. Kumar, S.; Stecher, G.; Tamura, K. MEGA7: Molecular Evolutionary Genetics Analysis Version 7.0 for Bigger Datasets. Mol. Biol. Evol. 2016, 33, 1870-1874. [CrossRef]

28. Conesa, A.; Götz, S. Blast2GO: A comprehensive suite for functional analysis in plant genomics. Int. J. Plant Genom. 2008, 2008, 1-12. [CrossRef]

29. Altschul, S.F.; Gish, W.; Miller, W.; Myers, E.W.; Lipman, D.J. Basic local alignment search tool. J. Mol. Biol. 1990, 215, 403-410. [CrossRef]

30. Wang, Y.; Li, J.; Paterson, A.H. MCScanX-transposed: Detecting transposed gene duplications based on multiple colinearity scans. Bioinformatics 2013, 29, 1458-1460. [CrossRef]

31. Larkin, M.A.; Blackshields, G.; Brown, N.P.; Chenna, R.; McGettigan, P.A.; McWilliam, H.; Valentin, F.; Wallace, I.M.; Wilm, A.; Lopez, R.; et al. Clustal W and Clustal X version 2.0. Bioinformatics 2007, 23, 2947-2948. [CrossRef] [PubMed]

32. Zhu, K.; Wang, X.; Liu, J.; Tang, J.; Cheng, Q.; Chen, J.-G.; Cheng, Z.-M. The grapevine kinome: Annotation, classification and expression patterns in developmental processes and stress responses. Hortic. Res. 2018, 5, 19. [CrossRef] [PubMed]

33. Shannon, P.; Markiel, A.; Ozier, O.; Baliga, N.S.; Wang, J.T.; Ramage, D.; Amin, N.; Schwikowski, B.; Ideker, T. Cytoscape: A software environment for integrated models of biomolecular interaction networks. Genome Res. 2003, 13, 2498-2504. [CrossRef] [PubMed]

34. Jiang, C.; Gu, X.; Peterson, T. Identification of conserved gene structures and carboxy-terminal motifs in the Myb gene family of Arabidopsis and Oryza sativa L. ssp. indica. Genome Biol. 2004, 5, R46. [CrossRef]

35. Ashburner, M.; Ball, C.A.; Blake, J.A.; Botstein, D.; Butler, H.; Cherry, J.M.; Davis, A.P.; Dolinski, K.; Dwight, S.S.; Eppig, J.T.; et al. Gene Ontology: Tool for the unification of biology. Nat. Genet. 2000, 25, $25-29$. [CrossRef]

36. Panchy, N.; Lehti-Shiu, M.; Shiu, S.-H. Evolution of gene duplication in plants. Plant Physiol. 2016, 171, 2294-2316. [CrossRef]

37. Dubos, C.; Stracke, R.; Grotewold, E.; Weisshaar, B.; Martin, C.; Lepiniec, L. MYB transcription factors in Arabidopsis. Trends Plant Sci. 2010, 15, 573-581. [CrossRef]

38. He, Q.; Jones, D.C.; Li, W.; Xie, F.; Ma, J.; Sun, R.; Wang, Q.; Zhu, S.; Zhang, B. Genome-wide identification of R2R3-MYB genes and expression analyses during abiotic stress in Gossypium raimondii. Sci. Rep. 2016, 6, 22980. [CrossRef]

39. Liu, H.; Xiong, J.; Jiang, Y.; Wang, L.; Cheng, Z.M. Evolution of the R2R3-MYB gene family in six Rosaceae species and expression in woodland strawberry. J. Integr. Agric. 2019, 18, 2753-2770. [CrossRef]

40. Li, H.-Y.; Yue, Y.-Z.; Ding, W.-J.; Chen, G.-W.; Li, L.; Li, Y.-L.; Shi, T.-T.; Yang, X.-L.; Wang, L.-G. Genome-wide identification, classification, and expression profiling reveals R2R3-MYB transcription factors related to monoterpenoid biosynthesis in Osmanthus fragrans. Genes 2020, 11, 353. [CrossRef] 
41. Liu, C.; Xie, T.; Chen, C.; Luan, A.; Long, J.; Li, C.; Ding, Y.; He, Y. Genome-wide organization and expression profiling of the R2R3-MYB transcription factor family in pineapple (Ananas comosus). BMC Genom. 2017, 18, 503. [CrossRef] [PubMed]

42. Du, H.; Yang, S.-S.; Liang, Z.; Feng, B.-R.; Liu, L.; Huang, Y.-B.; Tang, Y.-X. Genome-wide analysis of the MYB transcription factor superfamily in soybean. BMC Plant Biol. 2012, 12, 106. [CrossRef] [PubMed]

43. Starr, T.K.; Jameson, S.C.; Hogquist, K.A. Positive and negative selection of T cells. Annu. Rev. Immunol. 2003, 21, 139-176. [CrossRef] [PubMed]

44. Hanada, K.; Zou, C.; Lehti-Shiu, M.D.; Shinozaki, K.; Shiu, S.-H. Importance of lineage-specific expansion of plant tandem duplicates in the adaptive response to environmental stimuli. Plant Physiol. 2008, 148, 993-1003. [CrossRef] [PubMed]

45. Sharma, A.; Zheng, B. Molecular responses during plant grafting and its regulation by auxins, cytokinins, and gibberellins. Biomolecules 2019, 9, 397. [CrossRef]

46. Chen, Z.; Zhao, J.; Hu, F.; Qin, Y.; Wang, X.; Hu, G. Transcriptome changes between compatible and incompatible graft combination of Litchi chinensis by digital gene expression profile. Sci. Rep. 2017, 7, 3954. [CrossRef]

47. Shin, R.; Burch, A.Y.; Huppert, K.A.; Tiwari, S.B.; Murphy, A.S.; Guilfoyle, T.J.; Schachtman, D.P. The Arabidopsis transcription factor MYB77 modulates auxin signal transduction. Plant Cell 2007, 19, 2440-2453. [CrossRef]

48. Patzlaff, A.; McInnis, S.; Courtenay, A.; Surman, C.; Newman, L.J.; Smith, C.; Bevan, M.W.; Mansfield, S.; Whetten, R.W.; Sederoff, R.R.; et al. Characterisation of a pine MYB that regulates lignification. Plant J. 2003, 36, 743-754. [CrossRef]

49. Xu, W.; Tang, W.; Wang, C.; Ge, L.; Sun, J.; Qi, X.; He, Z.; Zhou, Y.; Chen, J.; Xu, Z.; et al. SiMYB56 confers drought stress tolerance in transgenic rice by regulating lignin biosynthesis and ABA signaling pathway. Front. Plant Sci. 2020, 11, 785. [CrossRef]

50. Zhang, L.; Liu, B.; Zhang, J.; Hu, J. Insights of molecular mechanism of xylem development in five black poplar cultivars. Front. Plant Sci. 2020, 11, 620. [CrossRef]

51. Rogers, L.A.; Dubos, C.; Surman, C.; Willment, J.; Cullis, I.F.; Mansfield, S.D.; Campbell, M.M. Comparison of lignin deposition in three ectopic lignification mutants. New Phytol. 2005, 168, 123-140. [CrossRef] [PubMed]

52. Yuan, H.; Zhao, L.; Chen, J.; Yang, Y.; Xu, D.; Tao, S.; Zheng, S.; Shen, Y.; He, Y.; Shen, C.; et al. Identification and expression profiling of the Aux/IAA gene family in Chinese hickory (Carya cathayensis Sarg.) during the grafting process. Plant Physiol. Biochem. 2018, 127, 55-63. [CrossRef] [PubMed] 